\section{Cimetidine-induced erythema annulare centrifugum: no cross-sensitivity with ranitidine}

Skin eruptions may occur in up to $1.9 \%$ of patients receiving cimetidine, an incidence marginally higher than that found in patients receiving placebo. ${ }^{1}$ The eruption most commonly described is mild, non-specific, erythematous, and maculopapular. Severe reactions, including bullous erythema multiforme have, however, been reported." Erythema annulare centrifugum is a pruritic, erythematous eruption beginning as a papule and progressively enlarging at the margin while clearing at the centre. It has been reported occasionally to be associated with underlying malignancy, dermatophyte infection, or drug treatment, though usually no cause is found and the lesions eventually disappear spontaneously after months or years. ${ }^{34}$ We describe a patient in whom erythema annulare centrifugum complicated long-term cimetidine treatment.

\section{Case report}

A 69-year-old woman first presented in February 1980 with a three-year history of increasing diarrhoea, nausea, and vomiting and a weight loss of $7 \mathrm{~kg}$ over 12 months. Upper gastrointestinal endoscopy showed large gastric folds and severe erosive duodenitis in the first and second parts of the duodenum. A high basal gastric acid output did not increase with pentagastrin and persistently raised serum gastrin concentrations failed to suppress after secretin Zollinger-Ellison syndrome was diagnosed. Complete control of her symptoms and a gain in weight occurred after treatment with cimetidine $200 \mathrm{mg}$ thrice daily and $400 \mathrm{mg}$ at night.

Six months after starting cimetidine a skin eruption developed, initially in the inguinal region and axillae, but soon becoming more widespread with moderate to severe pruritus. Individual lesions were fixed and lasted days to weeks. The eruptions consisted of slightly infiltrated circular erythematous lesions varying from 0.5 to $5.0 \mathrm{~cm}$ in diameter. The lesions tended to flatten and clear in the centre while activity remained at the edge. There was no scaling of the lesions at any stage. The inguinal and axillary regions were the most severely affected, but the lesions also occurred on the buttocks and upper trunk.
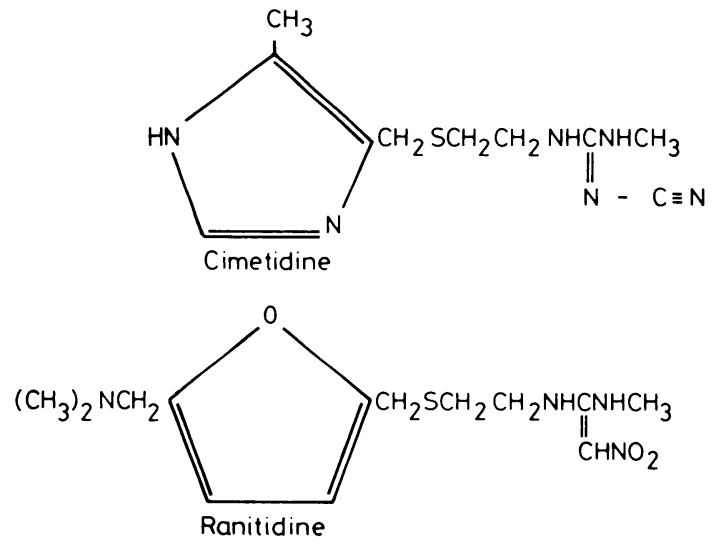

Comparative molecular structures of ranitidine and cimetidine.

Withdrawing cimetidine resulted in resolution of the eruption within three weeks, and treatment in that time consisted of simple emollients, including $1 \%$ menthol, $1 \%$ camphor in aqueous cream topically, and chlorpheniramine $4 \mathrm{mg}$ thrice daily by mouth. These were stopped when the eruption settled. Gastrointestinal symptoms recurred, however, and could not be controlled by high-dose antacid treatment combined with anticholinergics. Reinstituting cimetidine resulted in a return of the erythema annulare centrifugum within a week. Cimetidine was again discontinued and treatment with ranitidine $150 \mathrm{mg}$ twice daily was begun, with continued control of her gastrointestinal symptoms. The eruption resolved over three weeks and had not recurred six months later.

\section{Comment}

A skin eruption clinically consistent with erythema annulare centrifugum developed during the first six months of treatment with cimetidine for Zollinger-Ellison syndrome. That the eruption was directly related to cimetidine was supported by its resolution after withdrawal of the drug and its reappearance on rechallenge. There was no cross-reaction with ranitidine, another $\mathrm{H}_{2}$-receptor antagonist (figure), which probably reflected the differences in molecular structure between cimetidine and ranitidine.

${ }^{1}$ Kruss DM, Littman A. Safety of cimetidine. Gastroenterology 1978;74: 478-83.

2 Ahmed AH, McLarty DG, Sharma SK, Masawe AEJ. Stevens-Johnson syndrome during treatment with cimetidine. Lancet 1978;ii:433.

${ }^{3}$ Lazar P. Cancer, erythema annulare centrifugum, autoimmunity. Arch Dermatol 1963;87:246-51.

Shelley WB. Erythema annulare centrifugum due to Candida albicans. Br f Dermatol 1965;77:383-4.

(Accepted 6 fune 1981)

Gastroenterology Service and Department of Medicine, Alfred Hospital, Melbourne 3181, Australia

A C MERRETT, $M B$, BS, registrar, gastroenterology service

R MARKS, FACD, FRACP, senior lecturer in dermatology, Monash University, department of medicine

F J DUDLEY, MD, FRACP, director, gastroenterology service

\section{Herpes-zoster myelitis treated successfully with vidarabine}

Myelitis is a rare complication of herpes-zoster infections that may progress to severe paralysis and death. ${ }^{1}$ We report here a case of ascending myelitis after an attack of shingles in which there was a rapid improvement after treatment with vidarabine.

\section{Case report}

A 66-year-old man developed herpes zoster of the third and fourth thoracic (T3-4) dermatomes on the right. Two weeks later he noticed weakness of his right leg which caused him to drag the leg while walking. The weakness progressed so that his leg would give way while he was standing, and within a further two weeks he developed weakness of his right arm and hand. At this time he began to experience episodes of paraesthesia affecting his trunk and legs together with a more persistent numbness of his left leg.

He was admitted six wecks after his first symptom, and at this time he had a global weakness of his right arm (MRC grade 4) and right leg (grade 4 proximally, grade 2 distally). He had impaired light touch sensation below $\mathrm{T} 4$ on the right and impaired pain and temperature sensation below T6 on the left. Joint-position sense was impaired bilaterally at the toes, and vibration sense was impaired bilaterally below the sternum. Both knee and ankle jerks were brisk, and plantar responses were extensor. Haemoglobin concentration and white cell and platelet counts were normal and remained so throughout the illness. Radiography of the cervical and thoracic spine showed spondylitic changes. Findings on myelography were normal. Complementfixing antibodies to varicella zoster in the serum were $1 / 32$ (19 December 1980), 1;512 (5 January 1981), 1/1024 (20 January), 1/256 (2 February); in the cerebrospinal fluid they were $1 / 4$ (13 January), less than $1 / 2$ (20 January); cerebrospinal fluid protein concentration was $0.3 \mathrm{~g} / 1$, two lymphocytes $/ \mu 1$ (19 December 1980); cerebrospinal fluid protein concentration was $0.8 \mathrm{~g} / \mathbf{l}$, acellular (13 January 1981)

Deterioration continued after admission so that in the eighth week of his illness grip in the right hand became very weak (grade 2) and there was increased weakness in the right leg (grade 2 proximally, grade 0 distally). All modalities of sensation were now impaired bilaterally with a sensory level at T4 on the trunk, but in addition light-touch sensibility was impaired to the C6 dermatome on the right arm. Within a further week he had difficulty passing urine and was experiencing paraesthesia and numbness on the right side of his face. He was treated with vidarabine $(5 \mathrm{mg} / \mathrm{kg} /$ day $)$ for 10 days 10 weeks after the onset of his shingles. The treatment caused no side effects or haematological disturbances. Within three days of the start of the 